\title{
Quality of life
}

\section{Pancreas transplantation: assessing secondary complications and life quality}

\author{
P. S. Zehr, F. K. Milde, L. K. Hart and R. J. Corry \\ University of Iowa, lowa City, Iowa, USA
}

Summary. Two sequential studies of life quality among pancreas transplant recipients are reported. The first study $(n=32)$ investigated symptoms of neuropathy, enteropathy and retinopathy, along with well-being post successful transplantation. Patients noted improvement in secondary complications, were satisfied with the procedure and expressed hope for the future. The second study compared physical and social function, burden of symptoms, emotional/mental state and sense of well-being in successful $(n=31)$ and successful $(n=13)$ pancreas transplant recipients. Patient satisfaction with pancreas transplantation was high. Successful transplant patients perceived their health to be good, believed it would continue to be good, with life quality becoming better over time. The failed group expected life quality to become worse.

Key words: Pancreas - Transplantation - Diabetic Quality of life - Secondary complications

\section{Introduction}

Expert centres providing pancreas transplantation as a transplant option for patients with Type 1 (insulindependent) diabetes mellitus are experiencing improved graft and patient survival, as well as exceptional serum glucose control (Sutherland et al. 1991). Health care providers are able to look beyond the goal of improved patient survival for pancreas recipients, and consider the quality of the patient's social existence. Quality of life refers to " a person's subjective perceptions of his or her own well-being," (Jonsson 1987), and is related to the concepts of life-satisfaction, well-being, and happiness. Assessments of life quality are merging in the literature with objective assessments of diabetic symptomatology to support the efficacy of pancreas transplantation (Nakache et al. 1989; Zehrer et al. 1990; Voruganti et al. 1989). The purpose of this presentation is to summarize patient responses relative to their perceptions of secondary complications of diabetes, as previously published (Corry et al. 1990). Preliminary results of a comprehensive assessment of pancreas transplant recipients' perceptions of life quality will also be introduced.

\section{Subjects and methods}

In the first study, patients' perceptions of secondary complications were assessed using a questionnaire designed to compare pre-transplant and post-transplant symptomatology. The investigators were primarily interested in symptoms of retinopathy, neuropathy, and enteropathy, as well as the patient's mood and general well-being. Many of the patients were contacted personally or by telephone after returning the questionnaires to clarify their responses. All patients who received a functioning pancreas graft with resultant normal fasting blood sugars and glycosylated haemoglobin levels for 6 months or longer were eligible for the study. Thirty-two subjects responded and gave informed consent; 24 had received a combined renal-pancreas transplant while 8 had received a sequential pancreas following successful renal transplantation. All subjects had serum creatinine levels $<270 \mathrm{mmol} / 1$.

In the second study just completed, the quality of life of pancreas transplant recipients was investigated. A comprehensive questionnaire which incorporated 11 questionnaires was administered to assess physical and social function, burden of symptoms, mental state, and sense of wellbeing, Forty-four patients were interviewed after informed consent was obtained. Thirty one patients underwent successful transplantation and had normal blood glucoses, while the remaining 13 patients returned to insulin therapy following a failed graft. All of the recipients had received successful renal grafts as evidenced by serum creatinine levels $<270 \mathrm{mmol} / 1$. 
The data analysis for study one was limited to descriptive statistics. Since data collection in study two has just been completed, the statistical analysis of this preliminary report is limited to descriptive statistics and between group $T$-test comparisons of life quality. A more comprehensive analysis of the findings is forthcoming.

\section{Results}

\section{Neuropathy}

Patients were asked to recall if they experienced symptoms of neuropathy prior to their pancreas transplant. Twenty-one of the 32 patients responded positively. Of the 21 patients, 19 stated that these symptoms had improved or remained stable since the pancreas transplant. Of the remaining 11 patients who denied neuropathy symptoms pretransplant, 10 remained symptom-free. Therefore, 29 of 32 patients $(91 \%)$ stated that symptoms of neuropathy remained absent, remained stable, or improved post-pancreas transplant (Fig. 1a).

\section{Enteropathy}

Twenty-four of 32 patients experienced symptoms of enteropathy, i.e., nausea, vomiting, diarrhoea and constipation, prior to their pancreas transplant. Twenty-three of these 24 patients $(96 \%)$ stated that their symptoms had improved after the pancreas transplant. Of the total group of 32 patients, only 3 suggested progression of enteropathy symptoms (Fig. 1b).

\section{Retinopathy}

Statements regarding retinopathy were varied. Of the 27 patients with known retinopathy pretransplant, 8 patients felt that their vision had improved, 12 stated that their vision had stabilized, while 7 worsened. The 5 patients who denied retinopathy pre-transplant continued to have stable vision (Fig. 1c).

\section{Depression/Fatigue}

Twenty-one patients stated that they had an element of depression pre-pancreas transplant. While 3 of these patients continued to feel depressed, 18 of 21 (86\%) felt that their depression had improved. Two of the patients who denied depression prior to transplant felt depressed after transplantation (Fig. 2a).

Twenty-eight of 32 patients complained of fatigue pre-pancreas transplant. Twenty-six of these patients stated that their fatigue improved. Only one patient of the original 32 felt that their fatigue had worsened (Fig. 2b).

Preliminary results of the second study are as follows: The successful group had significantly higher perceptions of future life quality $(p<0.02)$, present health status $(p<0.009)$, and future health status $(p<0.009)$. They foresee a life of normal quality and getting better. The entire success group

\section{NEUROPATHY}

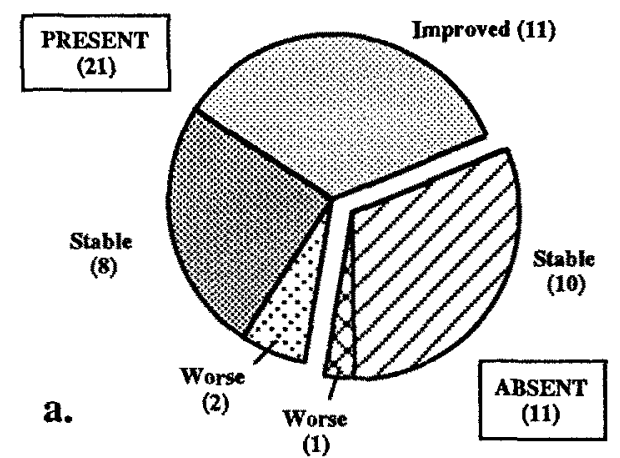

\section{ENTEROPATHY}

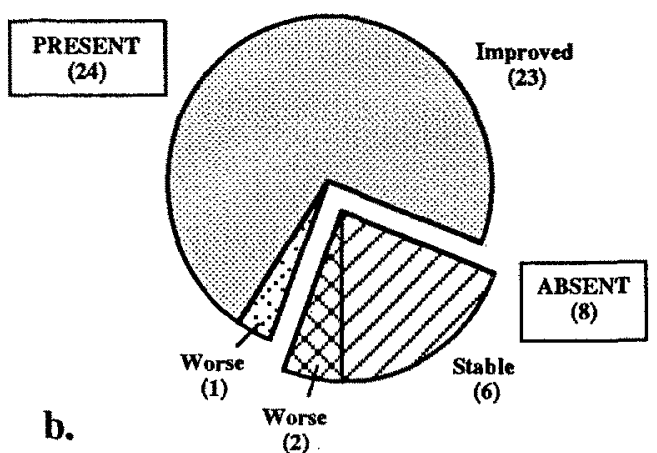

\section{RETINOPATHY}

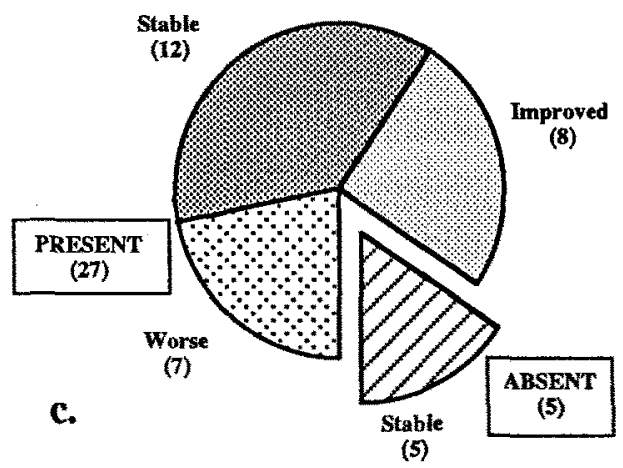

Fig. 1 The dotted section represents patients who experienced the complications pre-pancreas transplant. The lined section represents patients who denied the problem pre-pancreas transplant. The varying patterns (slices) represent how the problem is currently perceived. 
would undergo transplantation again, as would $46 \%$ of the failed group. The failed group expected life quality to become worse in the future. A more comprehensive presentation of these results is forthcoming.

\section{DEPRESSION}

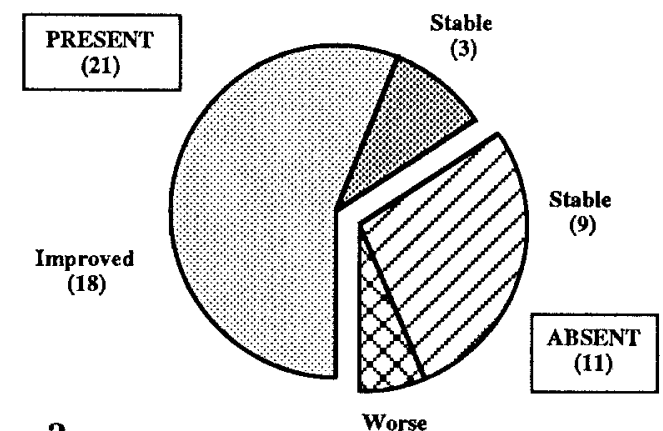

a.

(2)

\section{FATIGUE}

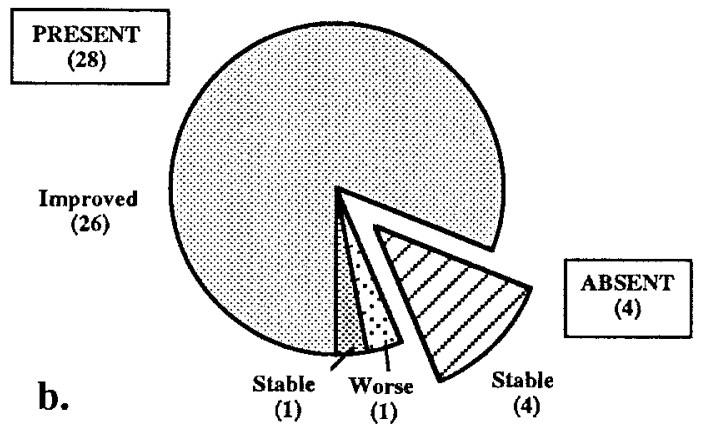

Fig. 2 The dotted section represents patients who experienced the complications pre-pancreas transplant. The lined section represents patients who denied the problem pre-pancreas transplant. The varying patterns (slices) represent how the problem is currently perceived.

\section{Discussion}

The efficacy of pancreas transplantation continues to be debated. Data emerging from the major centres, as presented at the 3rd International Spitzingsee Meeting, provides insight into the prevention or reversal of secondary complications of diabetes resulting from pancreas transplantation. It is clear from this study that patient satisfaction is high among this group, as the patients experience a reduced burden of diabetic symptoms. By reducing this burden, an improved life quality is anticipated.

The second study also strongly supports this statement; patient satisfaction with pancreas transplantation is high. It further suggests that successful patients perceive their health status to be good, and believe it will continue to be good, with life quality becoming even better over time.

Major pancreas transplant centres should continue in their efforts to merge data relevant to the status of secondary complications with quality of life measures. In this way, strong statements founded on research may be made supporting pancreas transplantation as therapeutic in appropriately selected diabetic patients.

\section{References}

Corry RJ and Zehr PS (1990) Quality of life in diabetic recipients of kidney transplants is better with the addition of the pancreas. Clin. Transplant. 4:238-241.

Jonsson B (1987) Assessment of quality of life in chronic diseases. Acta Paediatr. Scand. (Suppl) 337:164.

Nakache R, Tyden, G, and Groth C-G (1989) Quality of life in diabetic patients after combined pancreas-kidney or kidney transplantation. Diabetes 38 (Suppl. 1):40.

Sutherland DER, Gillingham K, and Moudry-Munns KC (1991) Registry report on clinical pancreas transplantation. Transplant. Proc. 23 (1):55-57.

Voruganti LNP, and Sells RA (1989) Quality of life of diabetic patients after combined pancreatic-renal transplantation. Clin. Transplant. 3:78-82.

Zehrer CL, and Gross CR (1990) Quality of life after pancreas transplantation. Diabetes Care 13 (5):539.

Pamela S. Zehr, RN, MA, CCTC

$1535 \mathrm{JCP}$

University of Iowa

Hospitals and Clinics

Iowa City, Iowa 52242

USA 